\title{
Kernos
}

Revue internationale et pluridisciplinaire de religion grecque antique

$26 \mid 2013$

Varia

\section{Marietta Horster, Anja Klöckner (éd.), Civic Priests. Cult Personnel in Athens from the Hellenistic Period to Late Antiquity}

\section{Stéphanie Paul}

\section{(2) OpenEdition}

1 Journals

\section{Édition électronique}

URL : http://journals.openedition.org/kernos/2142

DOI : $10.4000 /$ kernos. 2142

ISSN : 2034-7871

\section{Éditeur}

Centre international d'étude de la religion grecque antique

\section{Édition imprimée}

Date de publication : 10 octobre 2013

Pagination : 389-391

ISSN : 0776-3824

\section{Référence électronique}

Stéphanie Paul, « Marietta Horster, Anja Klöckner (éd.), Civic Priests. Cult Personnel in Athens from the Hellenistic Period to Late Antiquity », Kernos [En ligne], 26 | 2013, mis en ligne le 10 octobre 2013, consulté le 02 mars 2021. URL : http://journals.openedition.org/kernos/2142 ; DOI : https://doi.org/ $10.4000 /$ kernos. 2142

Ce document a été généré automatiquement le 2 mars 2021. 


\title{
Marietta Horster, Anja Klöckner (éd.), Civic Priests. Cult Personnel in Athens from the Hellenistic Period to Late Antiquity
}

\author{
Stéphanie Paul
}

\section{RÉFÉRENCE}

Marietta Horster, Anja Klöckner (éd.), Civic Priests. Cult Personnel in Athens from the Hellenistic Period to Late Antiquity, Berlin/Boston, De Gruyter, 2012. 1 vol. $16 \times 23,5 \mathrm{~cm}$, 249 p. (Religionsgeschichtliche Versuche und Vorarbeiten, 58). ISBN : 978-3-11-025807-3.

1 Le sujet des prêtres et des agents cultuels a connu ces dernières années un intérêt particulier au sein des études sur la religion grecque antique. Le projet de recherche dans lequel s'inscrit l'ouvrage ici recensé traite, plutôt que de la relation de médiation entre la communauté et leurs dieux (perspective verticale), de la position des prêtres au sein de ladite communauté (perspective horizontale). De manière plus spécifique, il se concentre sur la construction du rôle des prêtres et prêtresses d'un point de vue social, en explorant les interactions entre ces acteurs cultuels et les systèmes sociaux, culturels et politiques dans lesquels ils évoluent, du début de la période hellénistique jusqu'à la fin de l'Antiquité (p. 1). Cette thématique est destinée à être étudiée en deux volets géographiques distincts. Le présent volume, issu d'un séminaire tenu à Berlin en 2010, se concentre sur la documentation d'Athènes et de Délos; un second volume en cours de préparation sera consacré à l'Asie mineure et aux îles de l'Égée.

2 Une brève introduction des deux éditrices exposant la genèse et l'ambition du volume est suivie par une contribution de M. Horster établissant l'état de l'art et ouvrant de nouvelles perspectives d'étude sur le sujet. Elle insiste tout particulièrement sur l'importance de la prise en compte du contexte socio-politique. 
3 L'article d'A. Klöckner présente l'unique approche iconographique de l'ouvrage. Par une analyse précise et très fine, elle étudie la construction visuelle de l'image du prêtre à partir de deux reliefs votifs éleusiniens représentant le dédicant avec des divinités, une scène extrêmement rare aux époques hellénistique tardive et romaine. Le premier relief, érigé par un certain Lakrateidès, prêtre de Theos, Thea et Eubuleus pour luimême et sa famille, est daté de la fin $\mathrm{du}_{\mathrm{ii}}{ }^{\mathrm{e}}$ ou du début $\mathrm{du} \mathrm{i}^{\mathrm{er}} \mathrm{s}$. avant notre ère et était érigé dans le Plutonion d'Agélastos Petra à Éleusis. La scène représente le prêtre au milieu d'un cercle de divinités éleusiniennes. Le second relief, daté de la période antonine, est dédié selon l'inscription en hexamètre par un hiérophante du dème attique d'Hagnous aux déesses thesmophores. Le dédicant est représenté tourné vers Déméter trônant et Korè debout. Le contexte d'érection de ce relief diffère du premier, puisqu'il était sans doute placé au sein d'un espace sacré dans une maison privée. À partir de ces deux reliefs, l'A. étudie longuement la manière dont le recours visuel à la tradition peut servir de moyen de légitimation. Par ailleurs, elle montre que l'insertion des dédicants dans ce type de scènes souligne davantage la distance avec le divin qu'une éventuelle proximité. En effet, dans le premier cas, le prêtre est représenté « dans l'arrière-plan »: le relief de sa figure est moins prononcé que pour les autres et il n'entre pas en interaction avec les autres personnages. Dans le second cas, la distance se marque par le style différent des figures et la taille plus petite du dédicant. Ainsi, c'est la dignité de la fonction de la prêtrise, plutôt qu'une éventuelle relation individuelle avec le divin de ces deux personnages, que les reliefs veulent mettre en évidence.

4 C'est surtout sur la documentation épigraphique que se fondent les contributions suivantes. S. Lambert étudie la construction de la représentation des prêtres et prêtresses sur base d'un corpus de 28 décrets honorifiques émis par le Conseil et le Peuple d'Athènes du IV $\mathrm{e}$. avant notre ère jusqu'à la période augustéenne. Sur base de l'analyse de la formulation de ces décrets, S.L. aborde les thèmes de la spécificité de la fonction en rapport aux divers modes d'attribution et par rapport aux autres magistratures; la différence de représentation du point de vue du genre; l'évolution de la construction de ces représentations au cours du temps. L'A. constate ainsi que peu de différences distinguent les prêtrises gentilices des prêtrises dites "démocratiques ", ouvertes à l'ensemble des citoyens. Une exception, le cas le plus tardif (20/19 ap. J.-C.), qui souligne particulièrement l'eugeneia du dadouque Thémistoclès, est attribuée au contexte de la réforme de la citoyenneté athénienne qui a donné plus d'importance aux genè. En ce qui concerne la spécificité de la prêtrise par rapport aux autres magistratures, le langage des décrets n'est, une fois de plus, pas très explicite. L'objet de l'honneur porte le plus souvent sur l'action du prêtre dans la sphère religieuse, le sacrifice particulièrement, et sur ses contributions financières personnelles. La qualité d'eusebeia, qui souligne la relation envers les dieux, et la philotimia, un comportement davantage tourné vers la communauté, ne sont pas spécifiques à ces décrets. Quant au genre, même si quelques différences apparaissent (par ex. la mention du garant et l'absence de la philotimia), le langage épigraphique est bien moins explicite que l'iconographie, qui différencie davantage les rôles par le symbole du couteau sacrificiel, pour le prêtre, et de la clé, pour la prêtresse. L'A. rappelle en effet que ces décrets, proposés et votés par des hommes, appartiennent à une sphère proprement masculine. Sur base d'une étude diachronique, quelques évolutions sont perceptibles : la différence de moins en moins marquée entre les prêtres et les autres acteurs du culte dans l'objet des honneurs ou la mise en évidence croissante des contributions financières, qui va de 
pair avec un statut socio-économique des prêtres plus élevé. Cette importante étude se conclut par une annexe réunissant les textes pris en considération, accompagnés d'une traduction, qui constituera un outil précieux.

5 L'article d'E. Perrin-Saminadayar part du même type de documentation, mais se concentre sur une période plus restreinte, à savoir l'époque hellénistique tardive, un moment où Athènes a connu un regain de prospérité tant politique, économique que religieuse grâce à l'alliance avec Rome. L'apport de la prosopographie donne un éclairage complémentaire, tout en confirmant les conclusions de l'étude de S. Lambert : l'A. constate qu'il n'y a pas ou peu de différences entre les prêtrises et les autres magistratures, les premières s'insérant au même titre que les autres dans une carrière publique. L'A. conclut également à l'absence de différenciations entre les prêtrises d'Athènes et celles de Délos. Trois annexes s'ajoutent à l'article : 1) une liste des décrets constituant le corpus étudié; 2) une prosopographie des prêtres à Athènes et à Délos; 3) une reproduction des textes étudiés, sans traduction, qui pourra paraître redondante puisque ces mêmes textes se retrouvent dans l'annexe de S. Lambert (bien que le lemme et l'apparat critique soient ici un peu plus fournis).

6 M. Horster, dans une seconde contribution, s'attache aux implications politiques et religieuses de la prêtrise à travers deux aspects, le mode d'attribution et l'éponymie des prêtres. Concernant le premier, l'A. argumente que, contrairement à une opinion commune, le tirage au sort n'était pas particulièrement conçu dans les sources anciennes comme une méthode plus « démocratique » d'attribution de la prêtrise, pas plus que l'élection "oligarchique ». Elle traite également de la question de l'évolution dans l'attribution et la durée des prêtrises en regard de la perception romaine, plus aristocratique. En ce qui concerne l'éponymie des prêtres, deux cas retiennent l'attention: la prêtresse d'Athéna Polias et celle de Déméter et Korè à Éleusis. Il est remarquable que dans les deux cas et dans une certaine période de temps (hellénistique tardive et premier siècle de notre ère), les occurrences concernent surtout des jeunes officiants résidant dans le sanctuaire et sous la responsabilité de la prêtresse, situation où l'éponymie serait particulièrement appropriée.

7 Une brève contribution d'E. Sironen présente la documentation épigraphique et littéraire concernant les prêtrises attiques, au sens large, dans l'Antiquité tardive (III ${ }^{\mathrm{e}}$ $\mathrm{V}^{\mathrm{e}} \mathrm{s}$. de notre ère). L'étude de la représentation des prêtres à cette période est rendue difficile par le fait que la documentation se raréfie, surtout à partir de la seconde moitié du III $s$.

8 L'article de J.N. Bremmer, conçu à l'origine comme réponse aux communications de S. Lambert et de E. Sironen, sert de conclusion à l'ensemble du volume en revenant sur la définition du prêtre, du genre, et des changements perçus à l'époque augustéenne. C'est surtout sur l'Antiquité tardive que se concentre l'A., en prenant pour exemple la Vie d'Apollonios de Philostrate et les mystères d'Éleusis, et en concluant sur une comparaison entre les prêtrises païennes et chrétiennes.

9 En conclusion, l'ouvrage est d'une grande qualité dans ses contributions et d'une remarquable cohérence. Ainsi, une série de thématiques sont récurrentes telles que la construction du rôle du prêtre, la différenciation des genres - question encore peu explorée de manière systématique, et l'évolution de ce rôle au cours du temps. L'importance donnée à cette dernière problématique, ainsi qu'au contexte sociopolitique, est particulièrement souhaitable pour une période où les changements religieux doivent encore être mieux appréhendés. 


\section{AUTEURS}

STÉPHANIE PAUL

F.R.S.-FNRS - Université de Liège 